\title{
Prevalence of maxillary sinus alterations after zygomatic surgery. A comparative study between intra-sinus and ZAGA approaches
}

\author{
P.Clarós ${ }^{1}$, N. Końska ${ }^{2}$, P. Clarós-Pujol ${ }^{3}$, D. Clarós-Pujol ${ }^{3}$, J. Sentís ${ }^{4}$, A. Clarós ${ }^{1}$, M. Peñarrocha-Diago ${ }^{5}$, C. Aparicio ${ }^{6}$ \\ ${ }^{1}$ Clarós Otorhinolaryngology Clinic, Barcelona, Spain. \\ ORCID No: (Pedro Clarós 0000-0002-7567-0370, Andrés Clarós 0000-0001-6084-3470). \\ ${ }^{2}$ Department of Otorhinolaryngology, Stefan Zeromski Specialist Hospital, Cracow, Poland; Scholarship in Clarós Clinic, Barcelona, Spain. \\ ${ }^{3}$ Clarós Dental Clinics, Barcelona, Spain. \\ ${ }^{4}$ Department of Public Health (Statistics), School of Medicine, University of Barcelona, Barcelona, Spain. \\ ${ }^{5}$ Professor of Oral Surgery, Faculty of Medicine and Dentistry, University of Valencia, Spain. \\ ${ }^{6}$ Hepler Bone Clinic, ZAGA Center Barcelona, Spain.
}

*Corresponding author: Pedro Clarós, Clarós Otorhinolaryngology Clinic, Barcelona, Spain.

Received Date: April 26, 2021; Accepted Date: May 04, 2021; Published Date: May 10, 2021

Citation: P.Clarós, N. Końska, P. Clarós-Pujol, D. Clarós-Pujol, J. Sentís, et.al. (2021). Prevalence of maxillary sinus alterations after zygomatic surgery. A comparative study between intra-sinus and ZAGA approaches. J Dentistry and Oral Maxillofacial Surgery, 3(1); DOI: 10.31579/2643$6612 / 0018$

Copyright: (c) 2021. Pedro Clarós, This is an open access article distributed under the Creative Commons Attribution License, which permits unrestricted use, distribution, and reproduction in any medium, provided the original work is properly cited.

\begin{abstract}
Zygomatic implant (ZI) is an option of the edentulism. Complications associated with ZI are maxillary sinusitis and soft tissue recession. The Zygoma Anatomy-Guided Approach (ZAGA), reduce the complications of the Original zygomatic intra-sinus Surgical technique (OI-ST).
\end{abstract}

Study aims: 1) report and compare the prevalence of sinus complications between ZAGA and OI-ST. 2) propose therapeutic guidelines for sinus.

Material and methods: ZAGA has been proposed to reduce the complications of the OI-ST.

Results: Statistically significant increase of the "Modified Lund Mackay" (MLM) score (was noted in CT of patients after ZI surgery in comparison with the pre-surgical Lund-Mackay (LM) score ( $\mathrm{t}=5,0, \mathrm{p}<.0005)$.

Our study indicates that patients who underwent the ZI surgery according to the OI-ST. $(M=2.93, \mathrm{SD}=3.65)$ demonstrated a significantly higher ML-M score $(t=2.99, p<.003)$ compared to the patients operated with the ZAGA Concept $(M=1.52, \mathrm{SD}=2.36)$.

Conclusions: ZI procedures can lead to sinus alterations. Significant differences have been found between two different approaches.

Keywords: zygomatic implants; rhinosinusitis/surgery; Lund-Mackay score; zygoma anatomy-guided approach; edentulism; rehabilitation; antrostomy; zygomatic Brånemark technique;

Running title: Zygomatic Implants Techniques and Sinusitis

\section{Introduction}

Severe tooth loss and edentulism is one of the leading causes of disability in some high-income countries. Loss of natural tooth increases with age and, due to demographic changes (ageing population), is still a frequent condition, even with better dental care.

Tooth loss has a broad impact on general and psychological health. It limits an individual's capacity in biting, chewing, smiling and speaking; it distorts facial support and aesthetics, and leads to decreased self-esteem and lower quality of life.
Loss of masticatory pressure in areas of missing teeth is followed by the bone resorption of maxilla, first the alveolar process and afterward basal bone, which are a challenge in prosthetic rehabilitation. Lack of the alveolar bone prevents retention of a standard prosthesis, and the placement of conventional implants is impossible due to insufficient bone tissue for its anchorage.

There are several surgical techniques to treat severely resorbed maxilla with deficient alveolar bone, such as bone grafting and sinus augmentation. One of the therapeutic alternatives is the use of zygomatic implants. Zygomatic implants use zygomatic bone as the main support structure for implant fixation. Indications for rehabilitation with 
zygomatic implants are total or partial maxillary edentulism with extreme resorption (Fig. 1a, b), failure of conventional implant placement, failure of sinus augmentation or grafting procedures and rehabilitation after tumor resection and trauma [1].

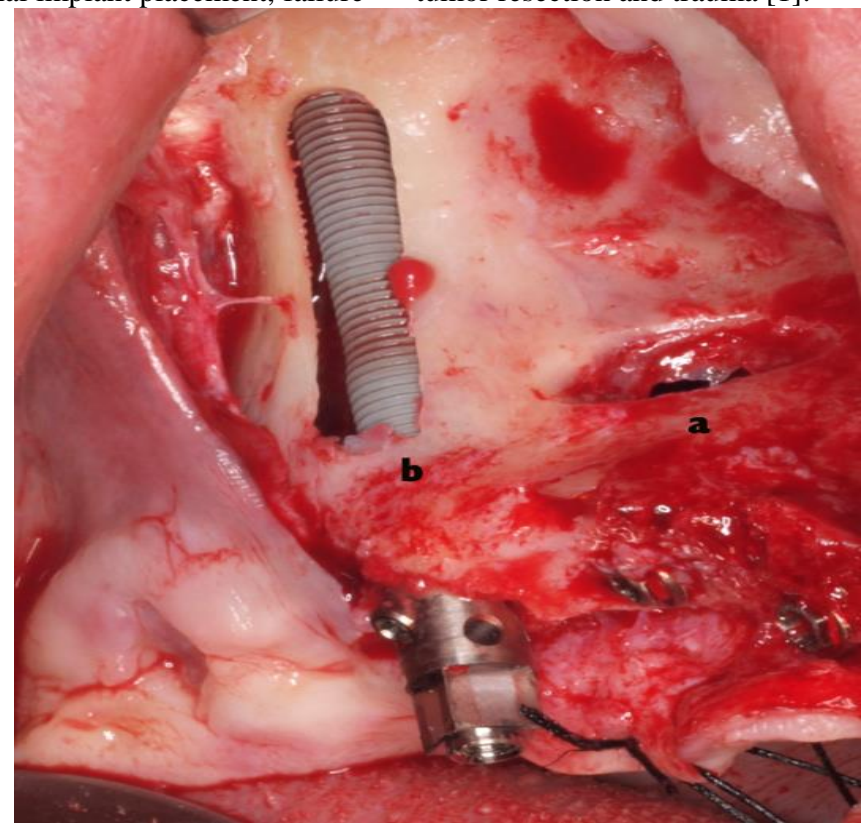

Figure 1. CBCT oblique cut of an extreme resorbed maxilla. The cut is representing the planned zygomatic implant position, first left molar, and direction (a); ZAGA implants were placed in positions of upper left canine and first molar $(b)$.

Recent studies have shown that the rehabilitation of atrophic jaws with the placement of zygomatic implants has higher success rates than bone reconstructions using large bone grafts from intra or extra-oral provenance [2].

Zygomatic implants were developed by Professor P. Brånemark. Initially implants had an intra-sinus path (OI-ST). Sinus entrance was performed from the palatal side; no care was taken about Schneider's membrane integrity $[1,2]$. A "window type" maxillary anterior wall antrostomy was performed at the superior level close to the zygomatic bone boundary to control zygoma drilling and the implant insertion path.
Surgery was performed in two stages. The greater the concavity of the anterior walls of the maxilla, the greater the palatal emergence of the implant head. Typical complications associated to the original method are late rhinosinusitis and bulky prostheses due to the palatal emergence of the implant head. In the year 2000, Stella and Warner proposed a modified method of implantation. In opposition to the "window type" osteotomy of the OI-ST, a slot type antrostomy was performed previous to implant placement along the foreseen future maxillary implant position. The implant is then placed through the crestal bone into the sinus, following the contour of the maxillary bone via a narrow slot until introducing the implant in the zygomatic process (Fig. 2).

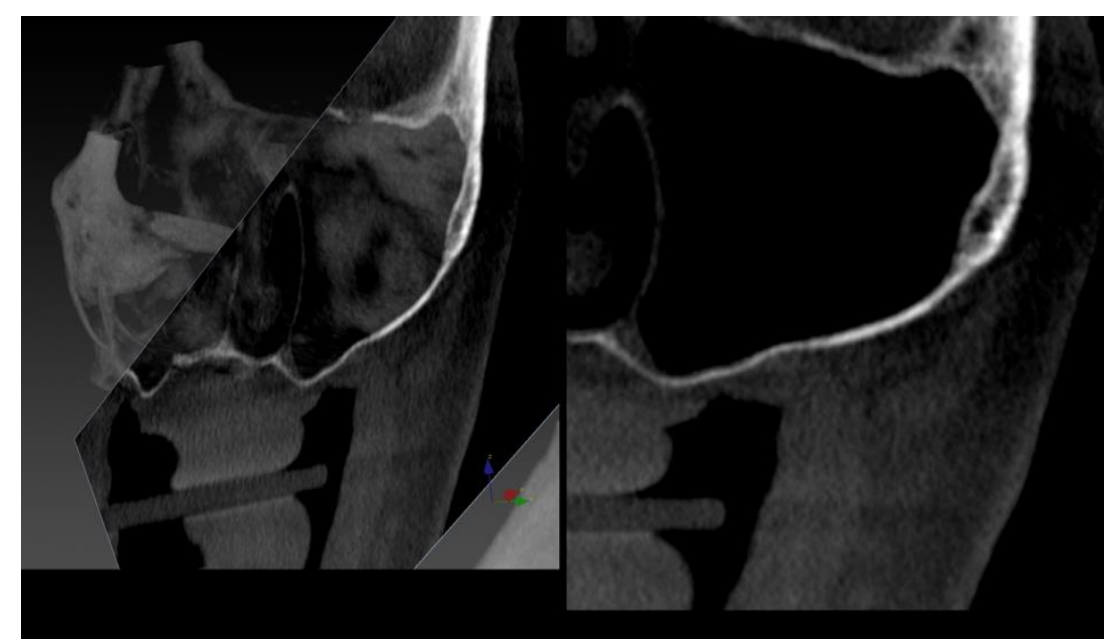

Figure 2. Typical slot made on the maxillary anterior wall previous implant placement. Note that the slot does not totally matches implant surface. Due to the perforation on the crestal bone, the slot technique is classified into the intra-sinus group

In this method detachment of the sinus membrane is not recommended [3, 4]. In 2006, the Aparicio group [5] described for the first time a one -year experience using a new approach for zygomatic surgery. In presence of concave maxillary wall that would lead to a bulky prosthesis, zygomatic implants were placed thorough the crestal bone. The implantation path was located partially outside of the maxillary wall. Neither window osteotomy, nor detachment of the sinus membrane was used. Almost simultaneously, the Brazilian group of Migliorança [6] 
named this approach as the Exteriorized technique. After a 3 year prospective study, Aparicio et al. [7] suggested that the extra-sinus approach eliminates the need for maxillary antrostomy or the creation of a slot previous to the surgery (Fig. 3a, b).

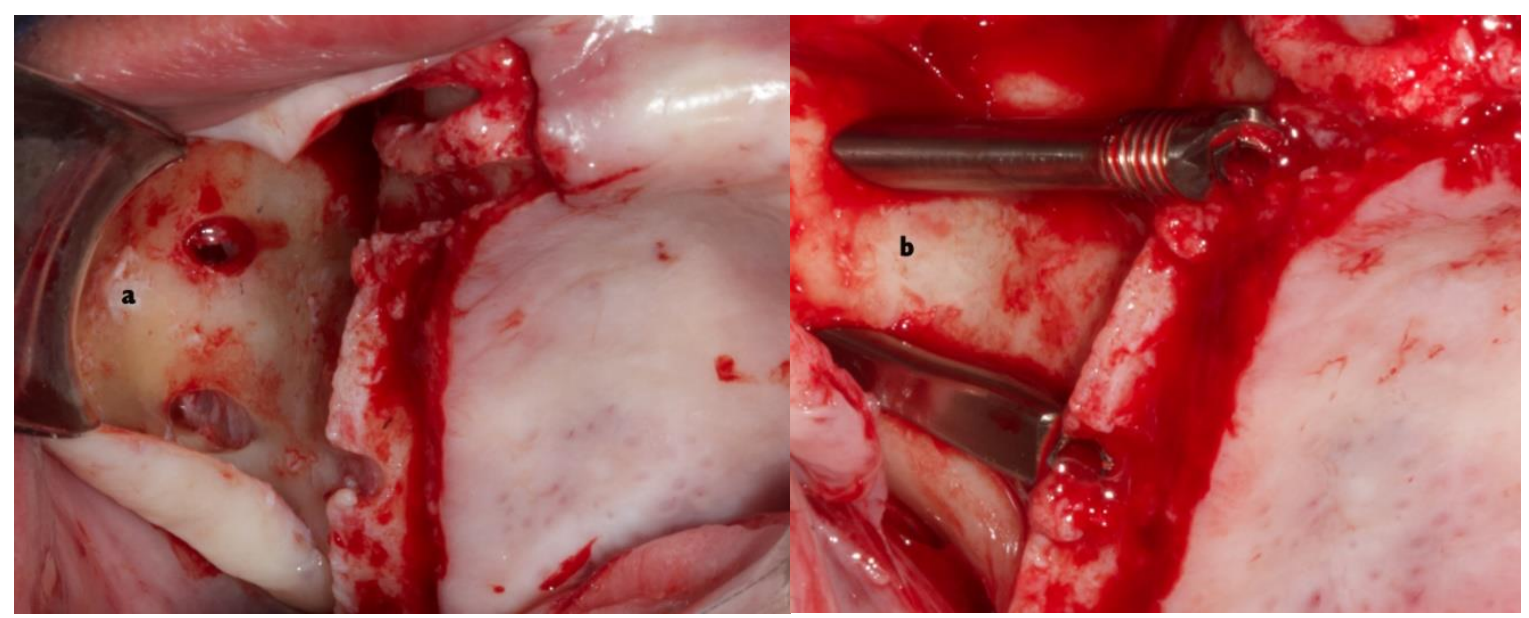

Figure 3. Example of the osteotomy performed on a patient showing a ZAGA type 4 anatomy. Integrity of the initial part of the membrane has been maintained (a); Implants in place. Both implants heads are placed on the crest. New designed ZAGA implants are capable to better adapt to the patient anatomy $(b)$.

The approach has fewer surgical stages than classic and sinus slot methods, is less invasive, reduces surgical time, and provides a shorter cantilever by the position of the implant emerging from the crestal bone at the level of the first molar region. The extra-maxillary technique didn't describe clear protocols of where to start the drilling, nor about the way the osteotomy should be performed $[8,9]$.

A relatively new concept named Zygomatic Anatomically Guided Approach (ZAGA) has been evaluated with promising results [10-12]. ZAGA is a development of the original zygomatic Brånemark technique, the Sinus Slot, and the extra-sinus approach, focused on inter-individual anatomical differences.

ZAGA Concept includes a variety of possibilities of implantation path according to the patient anatomy variations. If the residual alveolar bone at the sinus floor level has a minimum $4 \mathrm{~mm}$ height $\times 6 \mathrm{~mm}$ wide and the patient does not have a history of periodontitis, the crest bone entry point should be closed thorough the middle part of the crest with intra-sinus path of the implant (Fig. 4).

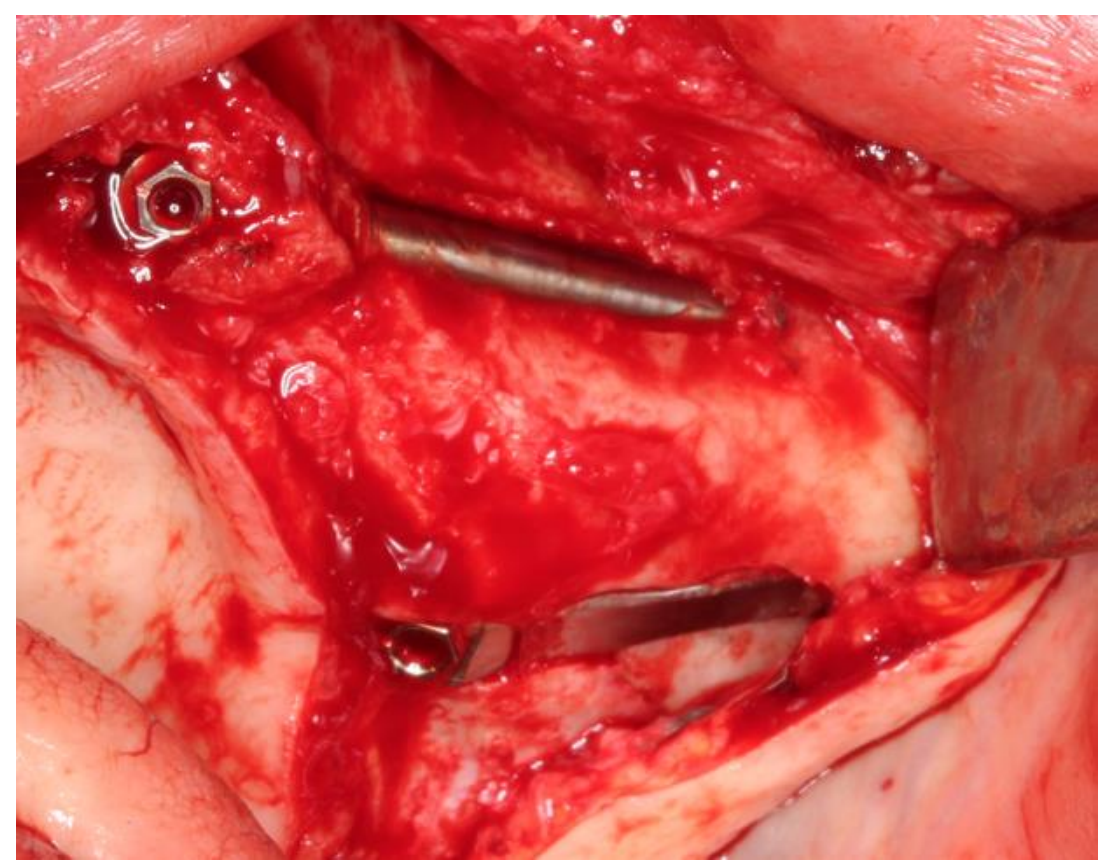

Figure 4. Example of ZAGA 0. Sinus floor height and wide are about 3,5 $\mathrm{mm}$ and $8 \mathrm{~mm}$ respectively. Implant path is planned to be intra-sinus. ZAGA type 0 is classified into the intra-sinus group. 
If, on the other hand, the bone height/thickness at the crest level is smaller or the anterior maxillary wall is more concave, the alveolar entry should be displaced towards the buccal area (Fig. 5 a,b).

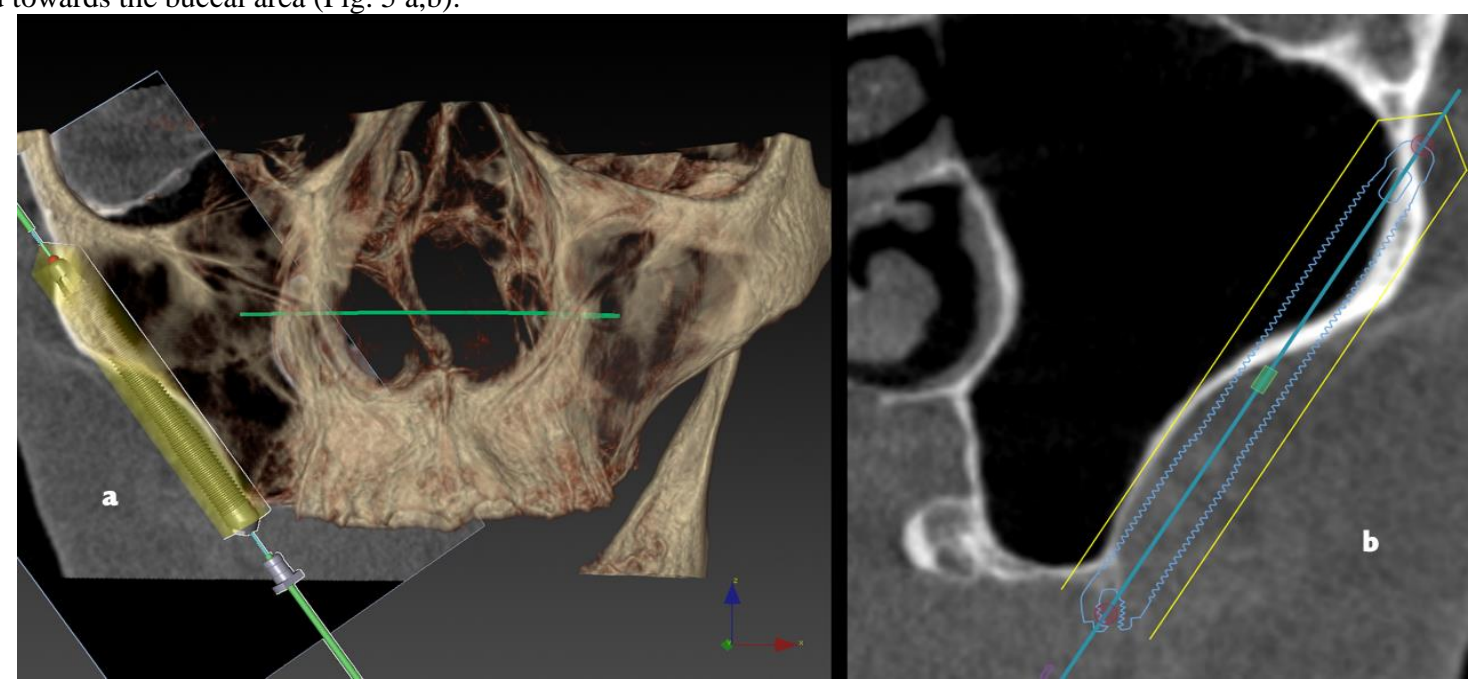

Figure 5. Virtual planning for zygomatic implant placement. The CBCT oblique cut is showing an extreme resorbed maxilla classified as ZAGA type 4 (a); CBCT oblique cut performed to the patient 3 years post-operatively. Note sinus status and implant positioning $(b)$.

The osteotomy will be in the shape of a tunnel or a canal depending on the height of the pristine bone and the concavity of the maxillary wall. Late sinusitis is reported to be the most common complication after the implantation of zygomatic implants [13, 14]. (Fig. 6)

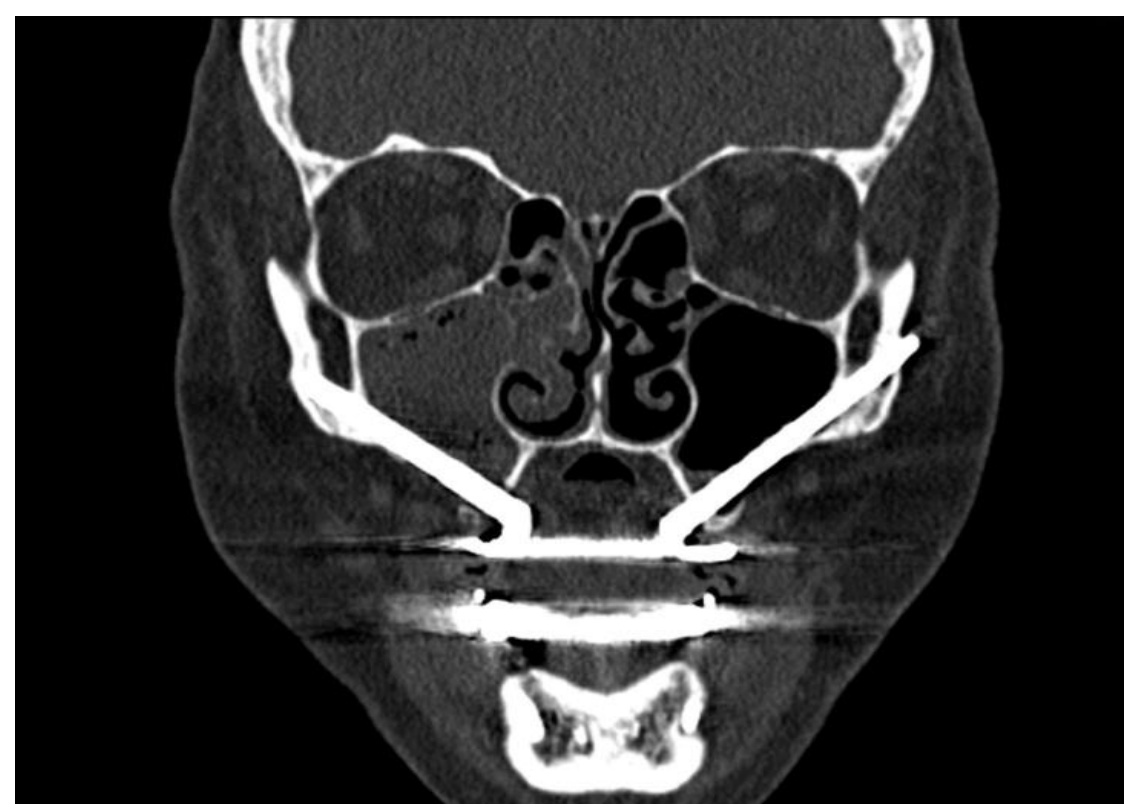

Figure 6. CBCT coronal cut showing two zygomatic implants placed using an intra-sinus path thorough the palatal aspect of the alveolar crest. Complete opacification of the right maxillary sinus affecting anterior ethmoidal sinuses can be observed.

The aim of this study is: 1) to report long-term outcomes in prevalence of sinus complications in patients rehabilitated using zygomatic implants; 2) to compare the prevalence of sinus complications depending on the type of surgical approach used; and 3) to propose diagnostic and therapeutic guidelines to treat sinus complications after placement of zygomatic implants.

\section{Materials and Methods}

Our retrospective study included 200 patients with severely atrophic edentulous maxillae restored with zygomatic implants with a follow-up at least 5 years after surgery. The surgeries were performed from 2004 to 2014 at the Aparicio Clinic, Clarós Clínic and others.
The patients were divided into two radiological groups depending on type of surgical procedure: the first group, Group 1, original zygomatic intra-sinus surgical technique (OI-ST), included 40 patients with 80 implants placed with the classical intrasinus approach, including those placed thorough a sufficient sinus floor bone, and the Slot technique. The second group, Group 2 included 160 patients treated with 320 implants placed according to the ZAGA Concept. In order to facilitate a non-biased radiological classification, patients from ZAGA Type 0, where an intrasinus path was chosen because the residual alveolar bone at the sinus floor level had at least $4 \mathrm{~mm}$ high x $6 \mathrm{~mm}$ wide, were excluded from the ZAGA Group. 
All patients included in the study had one preoperative and two postoperative exams with CT scan done immediately after the implant placement and the second at least 5 years after surgery. For most of the post-surgical Cone Beam Computer Tomography (CBCT) scans, a Kodak 9500 Cone Beam 3D System was used (Kodak, Carestream Health, Rochester, NY, USA). The images were obtained in the oblique-coronal, coronal, axial and horizontal planes, and cuts of $1.0 \mathrm{~mm}$ in thickness.

Each CBCT pre-operative scan was scored using the LundMackay (L-M) staging system for assessment of sinus abnormalities on tomographies.. Accordingly, the sinuses were divided into six groups: frontal sinus, anterior ethmoidal cells, posterior ethmoidal cells, maxillary sinus, sphenoid sinus and osteomeatal complex. Each group was given a score of 0,1 or 2 . Score 0 represented no abnormality or opacification thickness of sinus mucosa less than $3 \mathrm{~mm}$. A score of 1 indicated a partial opacification for the sinus with a mucosal thickening of more than $3 \mathrm{~mm}$; a score of 2 was given in cases of complete opacification of the sinus. The osteomeatal complex was assigned a score of either 0 (not obstructed) or 2 (obstructed). Each side was graded separately. A combined score ranged from 0 to 24 .

If the preoperative CT scan showed significant sinus alteration, in particular obstruction of osteomeatal complex, which plays a fundamental role in the development of rhinosinusitis, a functional endoscopic sinus surgery (FESS) was performed. Twenty-five patients (12.5\%) underwent a FESS operation before zygomatic implant placement. The surgeries consisted of an enlargement of the maxillary sinus ostium in an anterior or posterior direction with the inferior edge of the ostium untouched so as not to interfere with natural drainage patterns. The zygomatic implant surgery was postponed for a minimum of 6 months after the endoscopic sinus surgery, and a CT scan was conducted to check sinus status and to confirm the effectiveness of the operation.

\section{Statistical analysis}

The analysis was made using IBM SPSS Statistics 20.0 software. Frequencies and percentages were used to summarize qualitative data. Quantitative data was described using means and standard deviations, indicating the highest and the lowest score. To compare qualitative data between two groups, the Chi-Square test was used, and to compare means of independent quantitative data, the t-student test was used.

The dependent $\mathrm{t}$-test was used to compare the means between two related groups (pre- and post- zygomatic implantation) on the same dependent variable (L-M score).

The significance level used in our study was $\mathrm{p}<.05$.

\section{Ethical Approval:}

Prior to starting the study, all the researchers fully revised the material (X Rays and CT-Scans) received and bearing in mind, not one of them carried information of any of the patients and there has been absolutely no contact with any of them, the ENT Department of Clarós Clínic waived the Ethical Approval.

\section{All participant data have been anonymized.}

\section{Results}

At the moment of the implant placement surgery, $63 \%$ of patients $(n=126)$ represented completely normal (negative) CT scan without any opacification in the sinuses, meaning that the L-M score was 0 . The remaining CT scans presented certain degree of opacification of the sinuses. The mean L-M score was 1.11 (1.79) points, with the maximum L-M score of 8 .

A T-student test was performed to compare the preoperative L-M score between the two groups of patients. The difference in radiological sinus alterations between patients who qualified for zygomatic implantation using two different surgical approaches is not significant $\left(\mathrm{M}_{1}=0.83, \mathrm{SD}_{1}\right.$ $=1.3$ vs $\left.\mathrm{M}_{2}=1.18, \mathrm{SD}_{2}=1.83, \mathrm{t}=1.13, \mathrm{p}>.05\right)$.

When comparing the pre with the postoperative CT scans, 112 (56\%) patients presented a L-M score of 0 points (negative result) illustrating decreased or constant value over time, and $88(44 \%)$ of patients represented an L-M score of 1 or more (positive result). From the group of patients with a positive CT scan 86 patients $(97,7 \%$ ) presented an increased degree of opacification in one or both maxillary sinuses (L$\mathrm{M}$ score for maxillary sinus 1 to 4 ). The maximum L-M score was 14 points, the mean $\mathrm{M}=1.8, \mathrm{SD}=2.71$.

The forty patients who underwent the zygomatic implantation according to the classical intrasinus and crestal entrance (Slot \& ZAGA Type 0) approaches $(M=2.93, \mathrm{SD}=3.65)$, were compared to the 160 patients operated with the ZAGA Concept $(M=1.52$, SD $=2.36)$. Intrasinus group, demonstrated a significantly higher ML-M score $t=2.99, p$ $<.003$.

Pre- and post-operative L-M scores were found to be positively correlated, $(r=.7, p<.0005)$ in the entire studied population, and also in the Group $1(\mathrm{r}=.532, \mathrm{p}<.0005)$ and Group $2(\mathrm{r}=.835, \mathrm{p}<0005)$ when studied separately. This suggests that the normal (healthy) pre-surgical sinus condition is crucial in avoiding further sinus complications.

Of the twelve patients ( $6 \%$ of total 200 patients) who developed massive maxillary sinus alterations and obstruction of OMC underwent FESS, 8 of them belonged to Group 1 (intrasinus technique) and 4 patients belonged to Group 2 (ZAGA Concept). A Chi-Square test of independence was performed to examine the relation between type of surgical approach and the necessity of sinus surgery in patients after zygomatic implantation. The relation between these variables was significant, $\chi 2=17,4 \mathrm{p}<.0005$. Patients from the Group 1 were more likely than patients from the Group 2 to undergo FESS.

Two patients from Group 1 had an oro-sinusal fistula and massive sinusitis with peri-implant infection. Two implants (in 2 different patients) were removed despite pharmacological and surgical treatments.

A T-student test was performed to examine the relation between zygomatic implant surgery and tomographic alterations in sinuses. A statistically significant increase of the L-M score was noted in CT scans of patients after zygomatic implant surgery in comparison with the presurgical L-M score $(t=5,0, p<.005)$ in the entire studied population.

Moreover, when the 2 groups of operated patients were evaluated separately, a statistically meaningful increase in the L-M score was noted in the group operated using the classical intrasinus approach $t=4,3$, $p<.0005$, and also for the extra sinus group $t=3,3 p<.001$.

\section{Discussion}

Several authors have reported that rhinosinusitis can be a problem associated with zygomatic implants that emerges even after a long period of follow-up $[13,14]$. Currently, there is no consensus on how to report rhino-sinus status after zygomatic implantation. Actually, the term used to describe the sinus pathology in most of the studies about ZI, is "sinusitis", without clarifying the type, the associated signs and symptoms, or whether a CT scan or endoscopy was performed to confirm the diagnosis.

Our research outcomes showed a statistically meaningful increase in radiographic evidence of sinusitis in patients that underwent zygomatic implant surgery. The present results are consistent with data from previous studies [12].

The correlation between implantation of zygomatic implants and inflammation of the sinuses was reported by Aruajo et al. in 2017[15]. 
Between the years 2007-2014, 129 implants were placed in 37 patients using the sinus slot technique. Sinusitis was the most prevalent complication (8 patients, $21.4 \%$ ), which occurred from 10 months to 5 years postoperatively, although no clear diagnosis criteria was mentioned (clinical/ radiological). Five of the 8 studied patients were treated with antibiotic-therapy and the other 3 required maxillary antrostomy associated with antibiotics. Following the treatment, one patient shown recurrent sinusitis and the others had no new episodes.

D'Agostino et al. (2015) [16] analyzed 41 patients with 133 zygomatic implants placed (106 with intrasinus and 27 with extra sinus method) in years 2005-2014. Diagnosis of the sinusitis was based on clinical symptoms, which were evaluated using the Sino-Nasal Outcome Test-20 (SNOT-20) and the radiological findings were evaluated on CT scans using the Lund-Mackay Staging System. Radiologic evidence of sinusitis was found in $45.3 \%$ and $11.1 \%$. The sinus symptomatology was $15.6 \%$ and $11.1 \%$ of evaluated patients treated using the intrasinus and extra sinus techniques, respectively.

In a controlled study from 2014, Aparicio at al. [12] compared 2 groups of patients: treated by implantation of zygomatic implants using the classical technique (22 patients with 41 implants) versus the zygomatic anatomy- guided approach (ZAGA- 80 patients with 157 implants). The results showed significantly lower Lund-Mackay scores for the ZAGA group $(2.38+/-3.86$ vs $0.56+/-1.26, \mathrm{p}=.042)$ and a statistically significant difference $(54.55 \%$ vs $76.25 \%, \mathrm{p}=.047)$ concerning the percentage of patients with no signs or symptoms of rhinosinusitis: Lanza and Kennedy test negative and Lund-Mackay score zero. They concluded that ZAGA is associated with lower risk of maxillary sinus-associated pathology [12].

Acute maxillary sinus infection is an absolute contraindication to zygomatic implant placement and chronic sinusitis is a relative contraindication. The obstruction of the osteomeatal complex plays a fundamental role in the development of rhinosinusitis. Opacification, no permeability, of the rhino-sinus ostium should be treated before zygomatic implantation. The treatments methods must follow the current recommendations for the treatment of rhinosinusitis, including functional endoscopic sinus surgery (FESS). The obstruction of the osteomeatal complex plays a fundamental role in the development of rhinosinusitis. The purpose of FESS is to remove any anatomical obstructions that prevent proper mucosal drainage.

Olarte et al. suggest in their study that performing an inferior meatal antrostomy as a prophylactic intraoperative manoeuvre in patients who require zygomatic implant surgery is an effective method in preventing postoperative sinusitis [17]. D'Agostino et al. studied 13 patients who had undergone placement of 4 zygomatic implants and concurrent middle meatal antrostomy with an endoscopic approach. Twelve months after surgery mucosal, hypertrophy was found in 3 maxillary sinuses (11.5\%). They concluded that preoperative endoscopic sinus surgery provides statistically significant improvement in the health of the rhino-sinus system after zygomatic implant placement [18]. In our opinion, prophylactic sinus surgery before zygomatic implantation on the sinuses without any symptoms of alteration is not beneficial. It is important to be aware that any sinus operation will change the internal sinus environment. In the normal condition, unlike the post-surgical sinus, bacteria, viruses, and other foreign material that enter the nasal cavity invade the maxillary sinus itself very infrequently. Furthermore, the natural drainage pathway of the maxillary sinus is toward the natural ostium, located in the anteromedial aspect of the sinus towards the middle meatus, and persists even after performing inferior meatal antrostomy [19].

The best way to avoid sinus complications after zygomatic implant surgery is meticulous, pre- and postoperative radiological and clinical examination and appropriate treatment of sinusitis. If preoperatively the CT scans do not present any abnormalities in the sinuses, we see no reason to perform any kind of surgical prophylactic. However, if the patient presents any sinus abnormality in the CT scans, it is recommended to be evaluated by an ENT specialist and choose an appropriate treatment setting, either surgical, pharmacological or both.

Becktor et al.[20] established that sinusitis in patients with zygomatic implants can be attributed to the communication from the oral cavity into the antrum created by the internal threaded abutment screw chamber of the zygomatic implant; or to the loss of osseointegration, bone to implant contact, at the marginal level in the palatal area and the functional loading resulting in transversal mobility of the long coronal part of the zygomatic implant, which could imply a higher risk of communication between the antrum and the oral cavity. This may suggest that oral bacteria reached the sinus following the resorption of the periimplant alveolar bone. One potential problem may therefore be the development of sinus pathology when bone, already atrophic, is subjected to further bone loss such as by peri-implantitis; diagnosis was odontogenic sinusitis of peri-implant origin and was resolved by removing the implant.

In the postoperative assessment, sinus reactions to zygomatic implants have to be evaluated. Many patients could be subject to subclinical alterations of the paranasal sinuses and may be predisposed to subsequent complications. If any abnormality in the sinuses occurs, it is required to decide about treatment options in order to avoid losing the implant.

The strengths of the study include a large population of 200 patients evaluated, a follow-up time of at least 5 years, division into 2 different groups depending on the surgical technique, and the standardized method of evaluation of the CT scans using the L-M score system. All in all, further studies are necessary to determine the correlation between radiological and clinical findings and to follow-up with patients with zygomatic implants who underwent sinus surgery to determine the recurrence rate of sinusitis.

\section{Conclusion}

Our research outcomes showed a statistically meaningful increase in radiographic evidence of sinusitis in patients after with zygomatic implant surgery. Zygomatic surgery can lead to sinus alterations. Significant increased prevalence of sinus symptoms has been found in the OI-ST with respect to the ZAGA Concept. For this reasons it is important to establish clear surgical planning, taking into account clear diagnostic and therapeutic guidelines for the prevention and treatment of sinus complications.

\section{Acknowledgements}

All authors have reviewed the article and agreed to the submission.

\section{Authorship Contribution}

The principal investigator of the research, Pedro Clarós (MD $\mathrm{PhD}$ ), states that he had full access to all data in the study and takes responsibility for the integrity of the data and accuracy of the data analysis.

In our research Pedro Clarós (Clarós Clinic, Barcelona, Spain), Natalia Końska (Clarós Clinic, Barcelona, Spain; Department of Otorhinolaryngology at Stefan Zeromski Specialist Hospital, Cracow, Poland), Pedro Clarós-Pujol (Clarós Dental Clinics, Barcelona, Spain), Daniel Clarós-Pujol, (Clarós Dental Clinics, Barcelona, Spain) conducted and were responsible for the data analysis and wrote the paper. Miguel Peñarrocha (University of Valencia, Valencia, Spain), provide critical feedback, document formatting. Joan Sentís (Department of Public Health 
(Statistics), School of Medicine, University of Barcelona, Barcelona, Spain) conducted the statistical analysis. Carlos Aparicio (Hepler Bone Clinic, Barcelona, Spain) supervised the project. All authors provided critical feedback and helped shape the research, analysis and manuscript.

\section{Conflict Of Interest}

The authors report no potential conflict of interest.

\section{Funding}

The authors report no financial and material support for the research and the work in the manuscript.

\section{References}

1. Brånemark, PI, Gröndahl, K, Öhrnell, LO, et al. (2004). Zygoma fixture in the management of advanced atrophy of the maxilla: technique and long-term results. Scandinavian Journal of Plastic and Reconstructive Surgery and Hand Surgery, 38 (2):70-85.

2. Davo R, Felice P, Pistilli R, et al. (2018). Immediately loaded zygomatic implants vs conventional dental implants in augmented atrophic maxillae: 1-year post-loading results from a multicentre randomised controlled trial. Eur J Oral Implantol, 11 (2):145-161.

3. Stella JP, Warner MR. (2000). Sinus slot technique for simplification and improved orientation of zygomaticus dental implants: A technical note. The International Journal of Oral and Maxillofacial Implants, 15 (6):889-893.

4. Peñarrocha M, Uribe R, García B,et al. (2005). Zygomatic implants using the sinus slot technique-clinical report of a patient series. Int J Oral Maxillofac Implants, 20:788-792.

5. Ouazzani W, Arevalo X, Sennerby L,et al. (2006). Zygomatic implants: a new surgical approach. J Clin Periodontol, (33): 128 .

6. Migliorança R, Ilg JP, Serrano AS, et al. (2006). Sinus exteriorization of the zygoma fixtures: a new surgical protocol. Implant News, 3:30-35 [in Portuguese]

7. 7-Aparicio C, Ouazzani W, Aparicio A, et al. (2010). Extrasinus zygomatic implants: Three-year experience from a new surgical approach for patients with pronounced buccal concavities in the edentulous maxilla. Clin Implant Dent Relat Res, 12:55-61. Epub 2008 Dec 3. PMID: 19076181

8. 8-Migliorança R, Coppede A, Dias Rezende RC, et al. (2011). Restoration of the edentulous maxilla using extrasinus zygomatic implants combined with anterior conventional implants: a retrospective study. Int J Oral Maxillofac Implants, 26(3):665-672.
9. Maló P, Nobre M de A, Lopes I. (2008). A new approach to rehabilitate the severely atrophic maxilla using extramaxillary anchored implants in immediate function: a pilot study. $J$ Prosthet Dent, 100:354-366.

10. Aparicio C. (2011). A proposed classification for zygomatic implant patient based on the zygoma anatomy guided approach (ZAGA): a cross-sectional survey. Eur J Oral Implantol. Autumn; 4(3):269-275.

11. Aparicio C. (2012). The Zygoma Anatomy-Guided Approach (ZAGA). In: Aparicio C, Editor. Zygomatic Implants. The Anatomy-Guided Approach. Berlin: Ed. Quintessence.

12. Aparicio C, Manresa C, Francisco K, et al. (2013). Zygomatic Implants Placed Using the Zygomatic Anatomy-Guided Approach versus the Classical Technique: A Proposed System to Report Rhinosinusitis Diagnosis. Clinical Implant Dentistry and Related Research, 16 (5):627-642.

13. Vashisht R, Bhalla HK, Prithviraj DR. (2014). From maxilla to zygoma: A review on zygomatic implants. Journal of Dental Implants, 4(1):44-47.

14. Molinero-Mourelle P, Baca-Gonzalez L, Gao B, et al. (2016). Surgical complications in zygomatic implants: A systematic review. Medicina Oral Patología Oral y Cirugia Bucal, 21(6):751-757.

15. Araújo R, Sverzut A, Trivellato A, et al. (2017). Retrospective Analysis of 129 Consecutive Zygomatic Implants Used to Rehabilitate Severely Resorbed Maxillae in a Two-Stage Protocol. The International Journal of Oral \& Maxillofacial Implants, 32 (2):377-384.

16. Dagostino A, Trevisiol L, Favero V, et al. (2016). Are Zygomatic Implants Associated with Maxillary Sinusitis? Journal of Oral and Maxillofacial Surgery, 74(8):1562-1573.

17. Olarte H, Gómez-Delgado A, Trujillo-Saldarriaga S, et al. (2015). Inferior Meatal Antrostomy as a Prophylactic Maneuver to Prevent Sinusitis after Zygomatic Implant Placement Using the Intrasinusal Technique. The International Journal of Oral \& Maxillofacial Implants, 30(4):862-867.

18. Dagostino A, Favero V, Nocini R, et al. (2019). Does Middle Meatal Antrostomy Prevent the Onset of Maxillary Sinusitis after Zygomatic Implant Placement? Journal of Oral and Maxillofacial Surgery, 77(12):2475-2482.

19. Geng X, Jianbo S, Guanxia X, et al. (2011). Management of Persistent Maxillary Sinusitis: The View from China. In Duncavage JA. Becker SS eds. The Maxillary Sinus, 197-201.

20. Becktor JP, Isaksson S, Abrahamsson P, et al. (2005). Evaluation of 31 zygomatic implants and 74 regular dental implants used in 16 patients for prosthetic reconstruction of the atrophic maxilla with cross-arch fixed bridges. Clin Implant Dent Relat Res. 7:159-165. 
Ready to submit your research? Choose Auctores and benefit from:

* fast, convenient online submission

* rigorous peer review by experienced research in your field

* rapid publication on acceptance

* authors retain copyrights

* unique DOI for all articles

* immediate, unrestricted online access

At Auctores, research is always in progress.

Learn more www.auctoresonline.org/journals/journal-of-heart-andvasculature 